\title{
The effects of mitochondrial dysfunction on energy metabolism switch by HIF-1 $\alpha$ signalling in granulosa cells of polycystic ovary syndrome
}

\author{
Jing Wang ${ }^{1,2}$, Xiaohua $\mathrm{Wu}^{1,2}$ \\ ${ }^{1}$ Department of Obstetrics and Gynecology, Hebei Medical University, Shijiazhuang, China \\ ${ }^{2}$ Department of Reproductive Medicine Centre, Shijiazhuang Obstetrics and Gynecology Hospital \\ (The Fourth Hospital of Shijiazhuang), Shijiazhuang, China
}

\begin{abstract}
Introduction: This study aimed to determine the effects of mitochondrial dysfunction on energy metabolism of granulosa cells (GCs) and the competence of oocytes in polycystic ovary syndrome (PCOS).

Material and methods: A total of 107 patients who underwent controlled ovarian hyperstimulation $(\mathrm{COH})$ were enrolled. The clinical outcomes of patients with and without PCOS under in vitro fertilisation-embryo transfer (IVF-ET) were compared. Human primary GCs were exposed to mitochondrial and glycolysis inhibitors. Then, the related indicators of mitochondrial activity and glycometabolism were compared with controls. The viability of GCs after mitochondrial inhibitors was also determined.

Results: In PCOS patients, the number of retrieved oocytes significantly increased, but the high-quality embryos, available embryos, and high-quality blastocyst formation obviously decreased $(\mathrm{p}<0.05)$. Furthermore, the mitochondrial membrane potential, adenosine triphosphate (ATP) content, mitochondrial DNA (mtDNA) copy number, and HIF-1 $\alpha$ mRNA levels in GCs decreased, while the levels of reactive oxygen species increased $(\mathrm{p}<0.05)$. Mitochondrial inhibitors reduced the mitochondrial function, but increased the HIF- $1 \alpha$, GLUT1, LDHA, and PFKP gene expression. Glucose consumption gradually increased at 24, 48, and 72 hours of GC culture after CCCP treatment, and the viability of cells tested by CCK-8 increased $(\mathrm{p}<0.05)$

Conclusion: GCs are dependent on mitochondrial respiration and glycolysis for energy provision. Mitochondrial dysfunction accompanied by abnormal glycolysis was observed in PCOS patients, which affects the switch of energy from metabolic to glycolytic. The failure of transformation to glycolysis and low HIF- $1 \alpha$ expression in GCs during the development of follicles might be correlated with the low oocyte competence of PCOS. (Endokrynol Pol 2020; 71 (2): 134-145)
\end{abstract}

Key words: polycystic ovary syndrome; granulosa cells; glycolysis; mitochondrial functions; hypoxia inducible factor $1 \alpha$

\section{Introduction}

Polycystic ovary syndrome (PCOS) is one of the most common female endocrine and metabolic disorders, which affects approximately $5-10 \%$ women at the reproductive age $[1,2]$. It is highly heterogeneously characterised by hyperandrogenism, polycystic ovaries, and chronic anovulation, and it is attributed to three in four ovulatory infertilities [3]. Meanwhile, oocytes collected from PCOS patients who underwent in vitro fertilisation (IVF) are often of poor quality [4], leading to a high cancelation rate and low fertilisation rate $[5,6]$. Several factors are known to affect oocyte quality and subsequent embryo development, such as nutrition, hormonal regulation, and environmental influence [7]. However, the pathophysiology of the poor oocyte quality of PCOS remains not fully understood.

Recent research suggests that disordered follicular development may be the core of the abnormalities that occur in the ovary in PCOS [8]. During follicle growth, granulosa cells (GCs) surrounding the oocytes play an active role in oocyte differentiation and regulation through the proliferation and production of energy source $[9,10]$. Oocytes have low glycolytic activity and preferentially utilise the provision of energy substrates from GCs by glucose uptake for energy homeostasis [11]. A dysregulation in these functions can lead to severe cellular damage, causing decreased rates of oocyte nuclear maturation and fertilisation [12-14]. Data from several studies suggest that GC dysfunction contributes to abnormal folliculogenesis in PCOS [15]. However, there are few reports on abnormal glycolysis. Patients with PCOS consistently suffer from sub-fertility or infertility, and the poor quality of oocytes may be the main cause. Studies have shown that oocytes have low glycolytic activity and glucose uptake through the surrounding cells; hence, GCs are vital for oocyte homeostasis. Furthermore, GC energy metabolic dysfunction affects oocyte maturation and contributes to abnormal folliculogenesis in PCOS [11]. 
Glycolytic activity contributes to the cellular proliferation of GCs under hypoxic conditions during follicle maturation [16]. Hypoxia inducible factor $1 \alpha$ (HIF- $\alpha)$, which is the master regulator of cellular response to hypoxia, serves as a critical mediator of the adaptive response of tissue to low physiological oxygen levels. Previous reports have indicated that under hypoxia conditions, cells switch to the oxygen-independent metabolic pathway, and produce adenosine triphosphate (ATP) using the glycolysis pathway $[17,18]$. This process is also essential for higher quality oocytes and embryonic development. Hypoxic conditions increase the expression of HIF-1 complex regulated genes and are associated with the activation of glycolysis and suppression of mitochondrial oxidative phosphorylation (OXPHOS), which lead to the induction of a metabolic switch from OXPHOS to glycolysis.

Metabolic disorders have been acknowledged as a frequent cause of classic PCOS [19]. It has been suggested that decreased mitochondrial $\mathrm{O}_{2}$ consumption and glutathione, together with increased reactive oxygen species (ROS), contribute to the mitochondrial dysfunction in PCOS patients [20]. In addition, the development potential of oocytes with low mtDNA copy numbers is significantly reduced, thereby reducing blastocyst formation, which is consistent with the common symptoms of PCOS patients, such as anovulation and infertility [21]. GC mitochondrial dysfunction also affects oocyte maturation and development in PCOS [20]. Furthermore, it has been shown that in patients with PCOS, elevated oxidative stress is involved in the pathophysiology of anovulation and is associated with hyperglycaemia and hyperandrogenaemia [20].

All the above results lead to the hypothesis that maybe there is a relationship between the aberrant energy metabolism of GCs and PCOS-related abnormal follicles. Hence, the mitochondrial function and glycolytic activity, and the expression of HIF- $\alpha$ in patients with and without PCOS, who underwent IVF treatment, were compared in order to explore the relationship between the disorder's metabolic switch and abnormal expression of HIF- $\alpha$ in aberrant follicles development. This is the first functional study of PCOS patient-derived GCs that has explored the abnormal mitochondrial activities and aberrant expression of glycolysis metabolic genes in GCs.

\section{Material and methods}

\section{Subjects}

The Ethics Committee of the Reproductive Centre of Shijiazhuang Obstetrics and Gynaecology Hospital approved the present study. In addition, written, informed consent was obtained from each patient.
Clinical data were retrospectively collected from PCOS patients and tubal factor infertility patients (non-PCOS) during their first cycle of in vitro fertilisation-embryo transfer (IVF-ET). A short-acting gonadotropin-releasing hormone agonist (GnRH-a) long protocol was employed in the pre-ovulatory phase at the Reproductive Centre of Shijiazhuang Obstetrics and Gynaecology Hospital between April 2018 and December 2018. A total of 39 patients with PCOS and 68 patients with tubal infertility were enrolled in the present study. The reproductive age ranged from 21 to 33 years.

The inclusion criteria were as follows:

- diagnosis of PCOS according to the Rotterdam criteria of 2003: rare ovulation or anovulation (cycles longer than 35 days or shorter than 26 days);

- high androgen clinical manifestations or hyperandrogenism; polycystic ovaries [12 or fewer $(2-9 \mathrm{~mm})$ follicles in each ovary identified by transvaginal ultrasonography];

- compliance with at least two of the above and exclusion of other diseases causing related symptoms;

- all non-PCOS patients had normal ovarian morphology and regular menstrual cycles with tubal factor infertility.

The exclusion criteria were as follows: autoimmune diseases, internal diseases, chromosomal abnormality, endocrine or metabolic diseases, including hyperprolactinaemia and endometriosis, uterine malformations, ovarian surgery or only one ovary, history of taking hormonal medicine in the past three months.

\section{Clinical tests and IVF cycle characteristics}

The serum basal follicle-stimulating hormone (FSH), basal oestradiol (E2), basal luteinising hormone (LH), basal testosterone (T), fasting plasma glucose (FPG) levels on the second/third day of the menstrual cycle (follicular phase) after 12 hours of fasting, and the daily human chorionic gonadotropin (hCG) were measured at the Clinical Analysis Service of our hospital.

The characteristics of the IVF cycles included the following: total amount of administered FSH, days of using GnRH agonist, days of Gn stimulation, total number of retrieved oocytes, number of $\mathrm{MII}$ oocytes, number of $2 \mathrm{PN}$, overall fertilisation rate, high quality embryo rate, available embryo rate, high quality blastocyst formation rate, and clinical pregnancy rate.

\section{Human follicular fluid and GC preparation}

Follicular fluid (FF) and mural granulosa cells (GCs) were collected, isolated, and pooled from PCOS and non-PCOS patients who underwent IVF-ET by controlled ovarian hyperstimulation $(\mathrm{COH})$. The GnRH agonist (Ferring, Germany) was applied for approximately 18 days, when the downregulation standard was reached (FSH $<5 \mathrm{mIU} / \mathrm{mL}, \mathrm{LH}<5 \mathrm{mIU} / \mathrm{mL}$, E2 $<50 \mathrm{pg} / \mathrm{mL}$, endometrial thickness $<5 \mathrm{~mm}$ ), and $\mathrm{Gn}$ was subcutaneously injected until the hCG day. Then, cumulus-oocyte complexes (COCs) were retrieved from aspirated follicles 36 hours after the hCG trigger under transvaginal ultrasound guidance. After the COCs were removed from the follicular contents under a stereomicroscope using a Pasteur pipette, the follicular fluid was collected and centrifuged at $450 \mathrm{~g}$ for three minutes. Then, the clear supernatant was stored at $-80^{\circ} \mathrm{C}$ for subsequent glucose, pyruvate, and OS level assessment. The GCs at the bottom of the Eppendorf tube were divided into two parts; one part was stored at $-80^{\circ} \mathrm{C}$ for the detection of gene expression related to cell energy metabolism, while another part of the GCs was cultured for mitochondrial function and glycolysis analysis. Then, the oocytes were graded and subsequently inseminated. Pronuclear (PN) scoring was performed 16-18 hours after insemination. Embryo quality was assessed before every embryo transfer, and a maximum of three embryos were transferred to all patients at approximately 48 hours (four-cell stage) after insemination.

\section{Granulosa cells culture}

The GCs were isolated and purified by Ficoll density gradient centrifugation combined with the attachment culture method. Briefly, 
GCs that precipitated at the bottom of the centrifugal pipe were re-suspended in $2 \mathrm{~mL}$ of phosphate-buffered saline (PBS) and centrifuged at $450 \times \mathrm{g}$ for three minutes to obtain a pellet. Then, these GCs were digested with trypsin-EDTA solution (Solarbio, Beijing, China), according to the manufacturer's instructions. Subsequently, the cell suspensions were centrifuged at $300 \times \mathrm{g}$ for three minutes, the supernatant was discarded, and GCs that precipitated at the bottom were re-suspended with $2 \mathrm{ml}$ of PBS. Consequently, the cell suspension was slowly added to the Ficoll liquid surface and centrifuged at $300 \times \mathrm{g}$ for 15 minutes. After centrifugation, these GCs were located at the interface between the upper mixed liquid and lower Ficoll liquid. Then, a pipette was used to gently absorb the cell layer. Afterwards, the solution was re-suspended with $2 \mathrm{ml}$ of PBS and centrifuged at $300 \times \mathrm{g}$ for three minutes. Subsequently, the supernatant was poured out and a pellet was obtained. Next, the red blood cells (RBCs) were removed using erythrocyte lysis buffer. Then, $2 \mathrm{~mL}$ of lysis was added to the pellet in the tube and was gently shaken. After 20 seconds, the suspensions were centrifuged at $150 \times \mathrm{g}$ for three minutes. Then, the GC mass was resuspended in $3 \mathrm{~mL}$ of culture medium (DMEM/F12, $100 \mathrm{IU} / \mathrm{mL}$ of penicillin, $0.1 \mathrm{mg} / \mathrm{mL}$ of streptomycin and $10 \%$ foetal calf serum; Thermo Fisher Scientific, Inc., Waltham, MA, USA) and transferred to a six-well culture dish (NEST, Shanghai, China) at approximately $2.5 \times 10^{5}$ cells/well for 24 hours at $37^{\circ} \mathrm{C}$ with maximum humidity, and under atmospheric conditions of $5 \% \mathrm{CO}_{2}$ and $95 \%$ air. After 24 hours, the whole solution was replaced, the unattached GCs were removed by gentle pipetting, and the morphology of the attached GCs were observed using an optical microscope and used for the experimental analysis. Cells were counted using a haemocytometer and seeded at densities of $2 \times 10^{4}$ cells/well in a 96-well culture dish for 24 hours. Then, these were exposed to carbonyl cyanide $\mathrm{m}$-chlorophenyl hydrazine (CCCP, $10 \mathrm{mM}$ ) and bromopyruvic acid (BA, 0.5 or $5.0 \mathrm{mM}$ ), respectively, for further study. CCCP, which is a glycolysis inhibitor, and BA were diluted in DMSO $(\times 2000)$. Then, a control medium was added at the same concentration as that of the DMSO.

\section{Mitochondrial function assay \\ Mitochondrial membrane potential (MMP)}

A lipophilic cation, 5,5',6,6'-tetrachloro-1,1',3,3'-tetraethylbenzimidazol carbocyanine iodide (JC-1), was used to assess the mitochondrial status in GCs. JC-1 changes its fluorescence reversibly from green (monomeric status) to red (multimeric status) with the change in MMP. Furthermore, JC-1 is rapidly taken up by normal polarised mitochondria to form JC-1 aggregates, and presents a red spectral shift that results in increased levels of red fluorescence emission. In GCs of PCOS, the MMP collapses, and the JC-1 that accumulates within the mitochondria declines. In these cells, JC-1 remains in the cytoplasm in its monomeric form, and subsequently reduces the red fluorescence. Briefly, after culturing for 24 hours, the attached GCs from non-PCOS and PCOS were used to detect MMP using a Mitochondrial Membrane Potential Detection Kit (Solarbio, Beijing, China). GCs in 96-well plates were washed with PBS and incubated with JC-1 working solution at $37^{\circ} \mathrm{C}$ in a $\mathrm{CO}_{2}$ incubator for 20 minutes. After rinsing twice with JC-1 staining solution, the MMP was monitored by a fluorescence spectrophotometer, and the relative MMP was calculated. After the GCs were attached, these were treated by the inhibitors of CCCP and BA at 20 minutes to detect MMP using the JC-1 Assay Kit mentioned above and a fluorescent microscope (Olympus, Tokyo, Japan).

\section{ATP content}

The ATP content of GCs cultured for 24 hours and collected from the two groups was measured using a Firefly Luciferase-based ATP Kit (Beyotime, Nanjing, China), according to the manufacturer's instructions. Briefly, GCs were cultured at $2 \times 10^{4}$ cells/well in 96-well plates for 24 hours, lysed in ATP lysis buffer (from the kit), and centrifuged at $12,000 \times \mathrm{g}$ for 10 minutes. Then, the superna- tants were mixed with the testing buffer, and the ATP concentrations were measured using a luminometer (the experiments were conducted in triplicate). After 20 minutes of treatment with CCCP and BA, inhibitor-treated GCs and control cells were used to test the ATP content with the kit, as previously described.

\section{Intracellular ROS production}

The 2,7-dichlorofluorescin diacetate (DCFH-DA; Solarbio, Beijing, China) is a cell permeable peroxide-sensitive fluorescent probe used to detect intracellular free radicals. Once DCFH-DA enters into the cells and hydrolyses to dichlorofluorescin (DCFH) in the presence of free radicals such as $\mathrm{H}_{2} \mathrm{O}_{2}$, and peroxides, DCFH is oxidised to the green fluorescent product dichlorofluorescein (DCF) and trapped in the cell compartment, which can be measured by a fluorescence spectrophotometer. The wavelength of the laser was $488-525 \mathrm{~nm}$ (excitation and emission, respectively).

In the present study, two GC groups were collected and incubated with DCFH-DA at $37^{\circ} \mathrm{C}$ for 20 minutes. Then, fluorescent DCF were measured using a fluorescence spectrophotometer, and the relative absorbance values that characterise the ROS content were measured. At 30 minutes of CCCP and BA exposure, inhibitor-treated GCs and control cells were collected and incubated with DCFH-DA at $37^{\circ} \mathrm{C}$ for 20 minutes. Then, the ROS content was measured using a ROS Detection Kit.

\section{DNA extraction}

Total DNA extraction from isolated GC fractions was carried out using a Tissue Genome DNA Extraction Kit (Germany, Shanghai, China), according to the manufacturer's recommendations. Lysis was achieved by incubation of the sample material in a proteinase K solution. Appropriate conditions for DNA binding to the silica membrane in the centrifugal adsorption columns were obtained by the addition of chaotropic salts and ethanol to the lysate. The binding process was reversible and specific to nucleic acids. Contamination was removed by washing with two different buffers. The genomic DNA was finally eluted in $90 \mu \mathrm{L}$ of elution buffer.

\section{Quantification of mtDNA}

The mean mitochondrial DNA (mtDNA) copy number in GCs was determined by real-time quantitative PCR (Q-PCR) using the SYBR green DNA intercalator on the Chromo4 System ${ }^{\circledR}$ (Bio$\mathrm{rad}$, Hercules, CA, USA) in a $20 \mu \mathrm{L}$ reaction volume, containing a final concentration of $0.4 \mu \mathrm{M}$ of each gene-specific primer and $1 \mu \mathrm{l}$ of template. The mitochondrial gene quantitative primers: ND1 forward 5'-cctagccgtttactcaatcct-3', and reverse, 5'-tgatggctagggtgacttcat-3'; nuclear gene primers: $\beta$-actin forward 5 '-tggcacccagcacaatgaa-3' and reverse 5'-ctaagtcatagtccgcctagaagca-3', as an internal control to quantitate the nuclear DNA in GCs. The reactions were performed as follows: initial denaturation at $95^{\circ} \mathrm{C}$ for 10 minutes, and 40 cycles at $95^{\circ} \mathrm{C}$ for 45 seconds, $54^{\circ} \mathrm{C}$ for one minute, and $72^{\circ} \mathrm{C}$ for 45 seconds. Each sample was run in triplicate. The level of mtDNA copy number was calculated using the delta $\mathrm{Ct}(\Delta \mathrm{Ct})$ of the average $\mathrm{Ct}$ of the mtDNA and nDNA $(\Delta \mathrm{Ct}=\mathrm{Ctm}$ $\mathrm{tDNA}-\mathrm{Ct} \beta$-actin). The relative level of the mtDNA copy number was calculated using the ${ }^{2-\Delta \Delta \mathrm{Ct}}$ method.

\section{Activity of oxidative stress markers in follicular fluid (FF)}

The granulosa cells were isolated from all aspirated FFs in PCOS and non-PCOS patients using gradient centrifugation. The levels of malondialdehyde (MDA) and superoxide dismutase (SOD) in FF were respectively measured by thiobarbituric acid (TBA) and the chemiluminescence method. The 8-OHDG in FF was quantitatively detected by enzyme-linked immunosorbent assay (ELISA; Jiancheng, Nanjing, China). 


\section{Glucose test assay}

The glucose levels of FF in PCOS and non-PCOS patients were measured using a glucose assay kit (Jiancheng, Nanjing, China), according to the manufacturer's protocol, before culture. After the GCs were attached, these were treated by the inhibitors of CCCP at 24, 48, and 72 hours. After exposure, cells were collected by centrifugation, and the supernatants were stored at $-80^{\circ} \mathrm{C}$ for the glucose test. The samples were measured with a microplate reader at an absorbance of $505 \mathrm{~nm}$. The tests were repeated three times, and the glucose consumption from the study group was calculated and compared to the cell-free original medium.

\section{Cell Counting Kit-8 (CCK-8) assay}

The cellular proliferative activity of adherent GCs treated by the inhibitors of CCCP and cultured for 24, 48, and 72 hours was examined using a CCK-8 kit (Shanghai, Beyotime, China), according to the manufacturer's instructions, in a 96-well plate. The assay was repeated three times.

\section{Pyruvate production assay}

The pyruvate concentrations of FF in PCOS and non-PCOS patients were measured at an absorbance of $450 \mathrm{~nm}$, according to manufacturer's protocol (Jiancheng, Nanjing, China). The experiments were conducted in triplicate.

\section{Real-time reverse transcript polymerase chain reaction (RT-PCR)}

Total RNA was extracted from GCs in the non-PCOS and PCOS group using TRIzol (Solarbio, Beijing, China). Then, the complementary DNA (cDNA) was synthesised using a RevertAid First Strand cDNA Synthesis Kit (Jierui, Shanghai, China). The mRNA expression of GLUT1/LDHA/PFKP and HIF-1a was measured by real-time RT-PCR using the Power SYBR@ Green PCR Master Mix (Jierui, Shanghai, China) and normalised by the expression of the $\beta$-actin gene. The primers were selected according to previous reports, which were as follows: GLUT1 forward, $5^{\prime}$-ccagctgccattgccgtt$3^{\prime}$ and reverse $5^{\prime}$-gacgtagggaccacacagttgc- $3^{\prime}$; LDHA forward, $5^{\prime}$-tgcacccagatttagggactgat- $3^{\prime}$ and reverse $5^{\prime}$-cccaggatgtgtagcctttgag- $3^{\prime}$, PFKP forward $5^{\prime}$-aggcgatggacgagaggagat- 3 'and reverse $5^{\prime}$-tgatggcaagtcgcttgtag- $3^{\prime}$; HIF-1 $\alpha$ forward $5^{\prime}$-catcagctatttgcgtgtgagga- $3^{\prime}$ and reverse $5^{\prime}$-agcaattcatctgtgctttcatgtc- $3^{\prime}$. The specificity of these primers was validated by dissociation curve analysis. The relative mRNA levels were expressed as $2^{-\Delta \Delta C t}$ values.

\section{Statistical analysis}

Data are presented as mean \pm standard deviation. Statistical differences were analysed via $t$-test for the two groups, and one-way analysis was performed to analyse three or more groups. Analyses were performed using GraphPad Prism software version 5 (GraphPad software, San Diego, CA). $p<0.05$ was considered statistically significant.

\section{Results}

\section{Characterisation of the clinical IVF cycle}

A total of 107 patients who underwent 107 periods of $\mathrm{COH}$ were enrolled in the present study. There were no significant differences in the patients' age or duration of infertility in these two groups. The discrepancy of endocrine and biochemical indicators accorded with the characteristic performance of PCOS. The levels of serum LH, P and T, the FBG, and the AFC were significantly higher in the PCOS group than in the non-PCOS group. However, on the premise of the consistency of $\mathrm{COH}$ schemes, and under the same Gn dosage and usage time, the number of retrieved oocytes in the PCOS group was significantly higher than that in the non-PCOS group. Nonetheless, the rate used for judging the quality of the embryo and its subsequent development viability, including the high-quality embryo rate, available embryo rate, and high-quality blastocyst formation rate, obviously decreased in PCOS $(p<0.01)$. In comparing the available embryo rate of IVF, it was found that embryos originating from PCOS had a significantly poorer developing competence, with a rate of $43.81 \%$, while $58.72 \%$ of non-PCOS embryos could develop to available embryos before embryo transplantation. The general information and specific data on the outcome of IVF-ET in these two groups are presented in Tables 1 and 2.

Table 1. Comparison of patients' characteristics in both groups $(x \pm s)$

\begin{tabular}{lccc}
\hline Parameters & Control & PCOS & p value \\
\hline Number of cases & 68 & 39 & 0.1452 \\
\hline Age $[$ years] & $29.46 \pm 0.3484$ & $28.59 \pm 0.4884$ & $<0.0001^{* * * *}$ \\
\hline BMl $\left[\mathrm{kg} / \mathrm{m}^{2}\right]$ & $22.45 \pm 0.4306$ & $26.09 \pm 0.5643$ & 0.99 \\
\hline Duration of infertility [years] & $3.5 \pm 1.8$ & $3.4 \pm 1.0$ & $0.0284^{*}$ \\
\hline Base-FSH $[\mathrm{mlU} / \mathrm{mL}]$ & $5.538 \pm 0.2688$ & $4.704 \pm 0.1717$ & $<0.0001^{* * * *}$ \\
\hline Base-LH $[\mathrm{mlU} / \mathrm{mL}]$ & $3.800 \pm 0.2716$ & $7.003 \pm 0.4948$ & 0.6157 \\
\hline Base-E2 $[\mathrm{pmol} / \mathrm{L}]$ & $34.64 \pm 2.497$ & $36.53 \pm 2.697$ & 0.8681 \\
\hline Base-P $[\mathrm{nmol} / \mathrm{L}]$ & $0.9115 \pm 0.3740$ & $0.8062 \pm 0.5196$ & $<0.0001^{* * * *}$ \\
\hline Base-T $[\mathrm{nmol} / \mathrm{L}]$ & $0.9838 \pm 0.03860$ & $1.406 \pm 0.1080$ & $0.031^{*}$ \\
\hline FPG $[\mathrm{mmol} / \mathrm{L}]$ & $5.096 \pm 0.05803$ & $5.486 \pm 0.2124$ & \\
\hline
\end{tabular}

PCOS — polycystic ovary syndrome; BMI — body mass index; FSH — follicle-stimulating hormone; E2 — oestradiol; LH — luteinising hormone; T — testosterone; FPG — fasting plasma glucose 
Table 2. In vitro fertilisation (IVF) outcome parameters in both groups $(x \pm s)$

\begin{tabular}{lccc}
\hline Parameters & Control & PCOS & p value \\
\hline Days of Gn & $13.03 \pm 0.2583$ & $12.90 \pm 0.4265$ & 0.7792 \\
\hline Total dosage of Gn [IU] & $2753 \pm 115.7$ & $2654 \pm 188.0$ & 0.6349 \\
\hline Days of HMG & $7.000 \pm 0.5951$ & $4.880 \pm 0.6666$ & $0.0268^{*}$ \\
\hline Days of GNRH-a & $17.67 \pm 0.3563$ & $19.07 \pm 0.6676$ & $0.0454^{*}$ \\
\hline E2 on hCG [pmol/L] & $3481 \pm 146.4$ & $3457 \pm 203.1$ & 0.9222 \\
\hline Retrieved oocytes & $13.74 \pm 0.6395$ & $15.92 \pm 0.8827$ & $0.0447^{*}$ \\
\hline Number of MII & $12.59 \pm 0.5716$ & $14.49 \pm 0.8271$ & 0.0555 \\
\hline 2PN of fertilisation & $10.01 \pm 0.4659$ & $11.51 \pm 0.7160$ & 0.0705 \\
\hline Top quality embryos & $5.118 \pm 0.3290$ & $5.154 \pm 0.5084$ & 0.9504 \\
\hline Available embryos & $6.971 \pm 0.3875$ & $7.026 \pm 0.5685$ & 0.9345 \\
\hline Fertilisation rate (\%) & 72.91 & 72.30 & 0.8163 \\
\hline High quality embryos rate (\%) & 51.40 & 45.07 & $0.0383^{*}$ \\
\hline Available embryos rate (\%) & 58.45 & 50.37 & $0.0038^{* *}$ \\
\hline High quality blastocyst formation rate (\%) & 14.18 & 10.09 & $0.0434^{*}$ \\
\hline Clinical pregnancy rate (\%) & 77.14 & 38.46 & $0.0176^{*}$ \\
\hline
\end{tabular}

PCOS — polycystic ovary syndrome; Gn — gonadotropin; E2 — oestradiol; hCG — human chorionic gonadotropin; HMG — human menopausal gonadotropin; $\mathrm{GnRH}-\mathrm{a}$ — gonadotropin-releasing hormone agonist; 2PN — two-pronuclear zygote; MII — metaphase II oocytes

\section{Mitochondrial membrane potential}

In the present study, GCs were collected from matured follicles of non-PCOS and PCOS ovaries undergoing ovarian stimulation for IVF, and the MMP was compared between these two groups. As shown in Figure 1, the MMPs of GCs were analysed using image-based cytometry. The MMP of GCs was significantly lower in PCOS, when compared to non-PCOS $(1.073 \pm 0.09$ vs. $0.785 \pm 0.04)$. In the cultured GCs, and in the treat- ment with mitochondrial inhibitors (CCCP, $10 \mu \mathrm{M})$ and glycolysis inhibitor (BA, $0.5 \mathrm{mM} / 2.5 \mathrm{mM}$ ), all had a significant effect on MMP. The intensity of red fluorescence gradually decreased, and the green intensity gradually increased. Finally, the relative values of MMP declined (control, $1.094 \pm 0.12$; CCCP, $0.476 \pm 0.07$; BA $0.5 \mathrm{mM}$, $0.508 \pm 0.18$; BA $2.5 \mathrm{mM}, 0.476 \pm 0.13$ ). The results confirm the reduction of red to green fluorescence by approximately one-half in response to $10 \mu \mathrm{M}$ of CCCP.

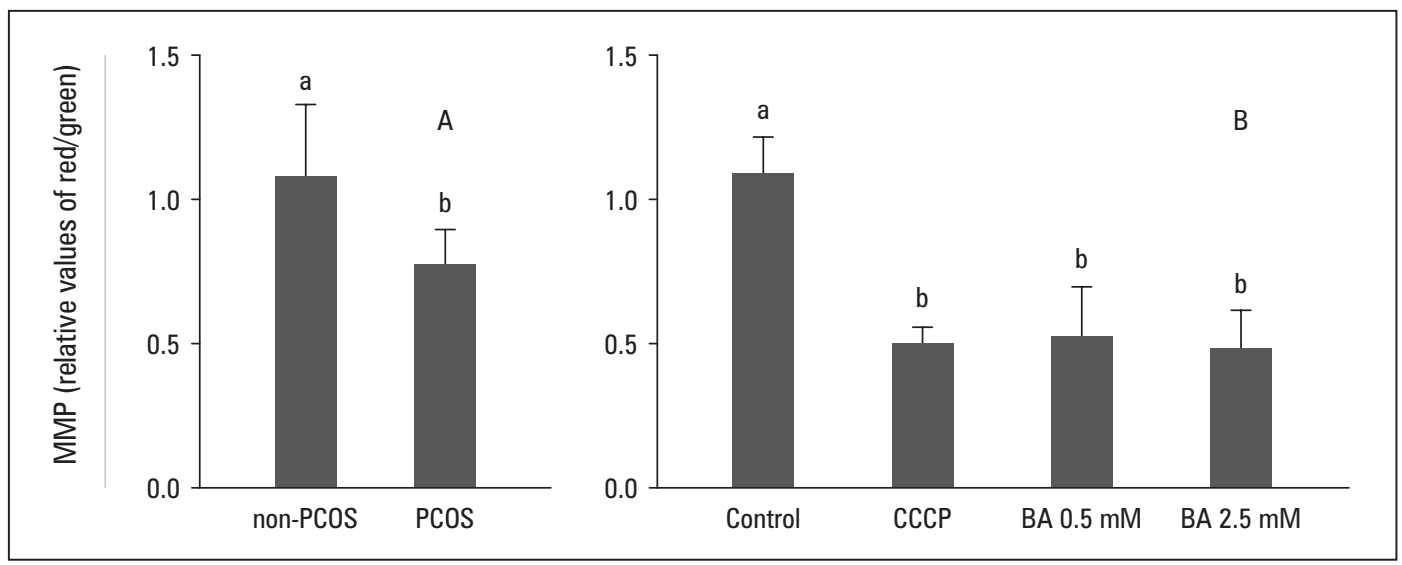

Figure 1. Mitochondrial membrane potential (MMP) in human granulosa cells (GCs) from patients without polycystic ovary syndrome (non-PCOS) and patients with polycystic ovary syndrome (PCOS), and the following treatment with inhibitors of mitochondrial function or glycolysis activity - image-based cytometry: mitochondrial membrane potential (MMP) was measured by the relative values of red to green fluorescence, as determined. MMP in GCs was compared between non-PCOS and PCOS. B) GCS were treated with CCCP $(10 \mu \mathrm{M}), B A(0.5 \mathrm{mM} / 2.5 \mathrm{mM})$ for $30 \mathrm{~min}$, and MMP in GCs was then measured by the ratio of red to green fluorescence intensity. Bars represent average values \pm SEM of MMP in $2 \times 10^{4}$ GCs. The t-test and one-way ANOVA were used for statistical analysis. $a-b, p<0.05$ 


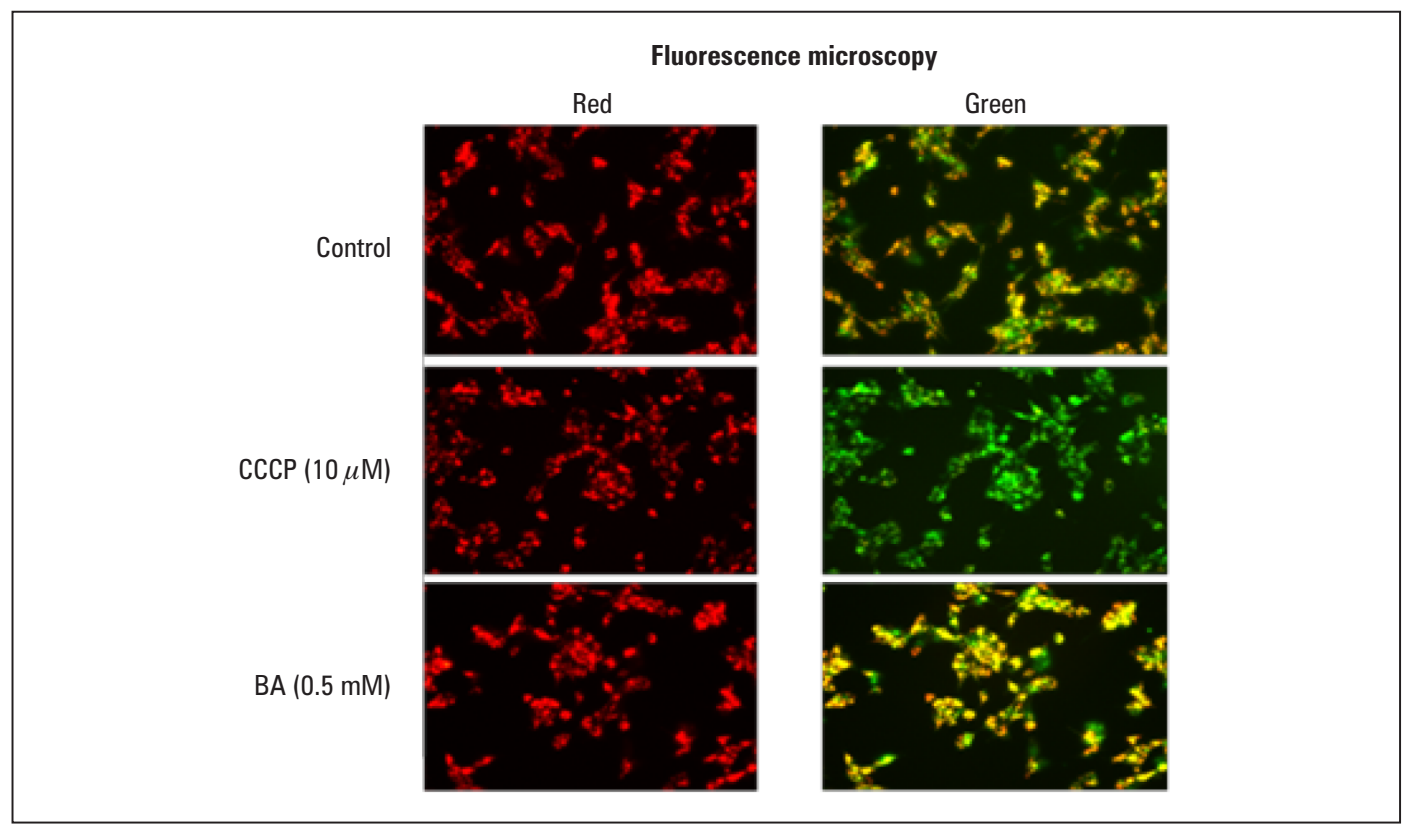

Figure 2. Mitochondrial membrane potential (MMP) in human granulosa cells (GCs) from patients without polycystic ovary syndrome (non-PCOS) and patients with polycystic ovary syndrome (PCOS), and the following treatment with inhibitors of mitochondrial function or glycolysis activity - fluorescence microscopy: GCs were treated with CCCP (10 $\mu \mathrm{M}), B A(0.5 \mathrm{mM})$ for $30 \mathrm{~min}$; as the MMP declined the intensity of red fluorescence decreased to green fluorescence

Therefore, $10 \mu \mathrm{M}$ of CCCP was used as the standard concentration in all further studies $(\mathrm{n}=3$, separate experiments).

As shown in Figure 2, the fluorescence microscopy revealed that the green fluorescence intensity in GCs increased when-GCs were treated with mitochondrial inhibitors and the glycolysis inhibitor, while the red fluorescence intensity diminished. In addition, the intensity of green fluorescence in CCCP-treated GCs was the most obvious, when compared to those treated with $B A(n=3$, in separate experiments).

\section{ATP content}

As shown in Figure 3, the ATP content of GCs was significantly lower in PCOS than in non-PCOS $(2.484 \pm 0.03$ $\mu \mathrm{m}$ vs. $3.916 \pm 0.09 \mu \mathrm{m}$ ). In addition, after the treatment

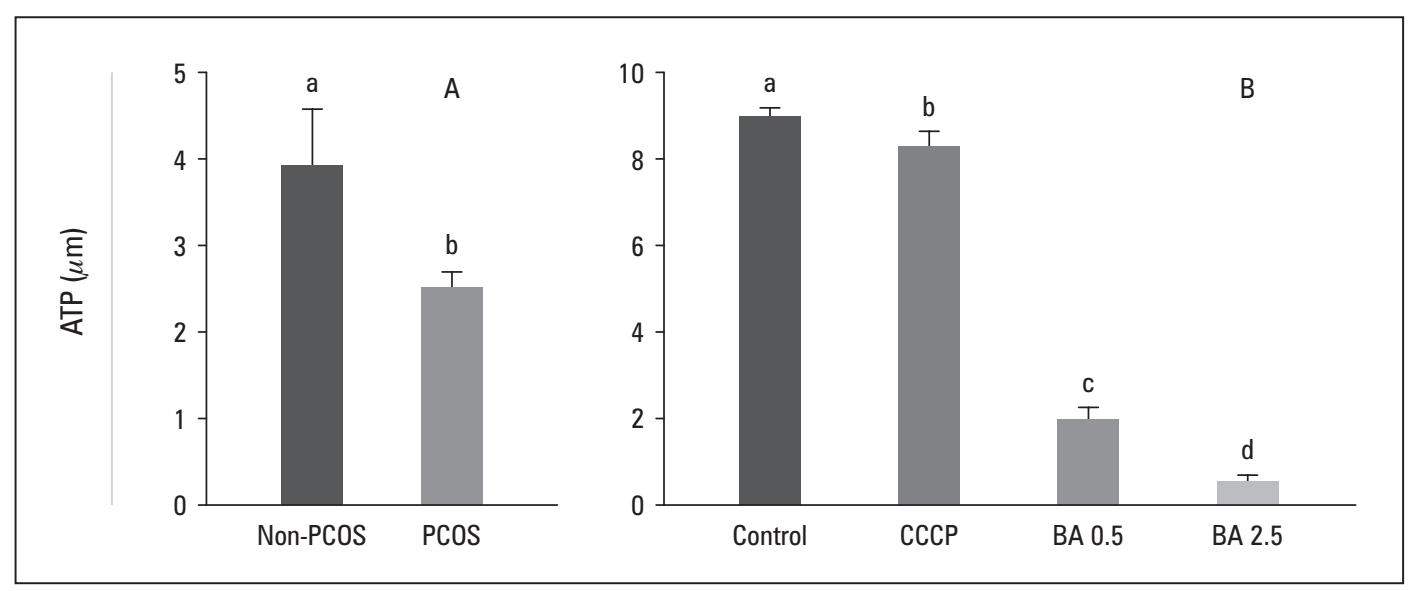

Figure 3. Adenosine triphosphate (ATP) content in human granulosa cells (GCs) from patients without polycystic ovary syndrome (non-PCOS) and patients with polycystic ovary syndrome (PCOS), following the treatment with inhibitors of mitochondrial function or glycolysis activity. A. ATP content in GCs were compared between non-PCOS and PCOS. B. GCs were treated with CCCP (10 $\mu \mathrm{M})$, $B A(0.5 \mathrm{mM} / 2.5 \mathrm{mM})$, or vehicle (DMSO, Control), respectively, for $30 \mathrm{~min}$, and ATP content was then measured. Bars represent average values $\pm S E M$ of ATP content in $2 \times 10^{4}$ GCs. The t-test and one-way ANOVA were used for statistical analysis $(a-d, p<0.05)$ 


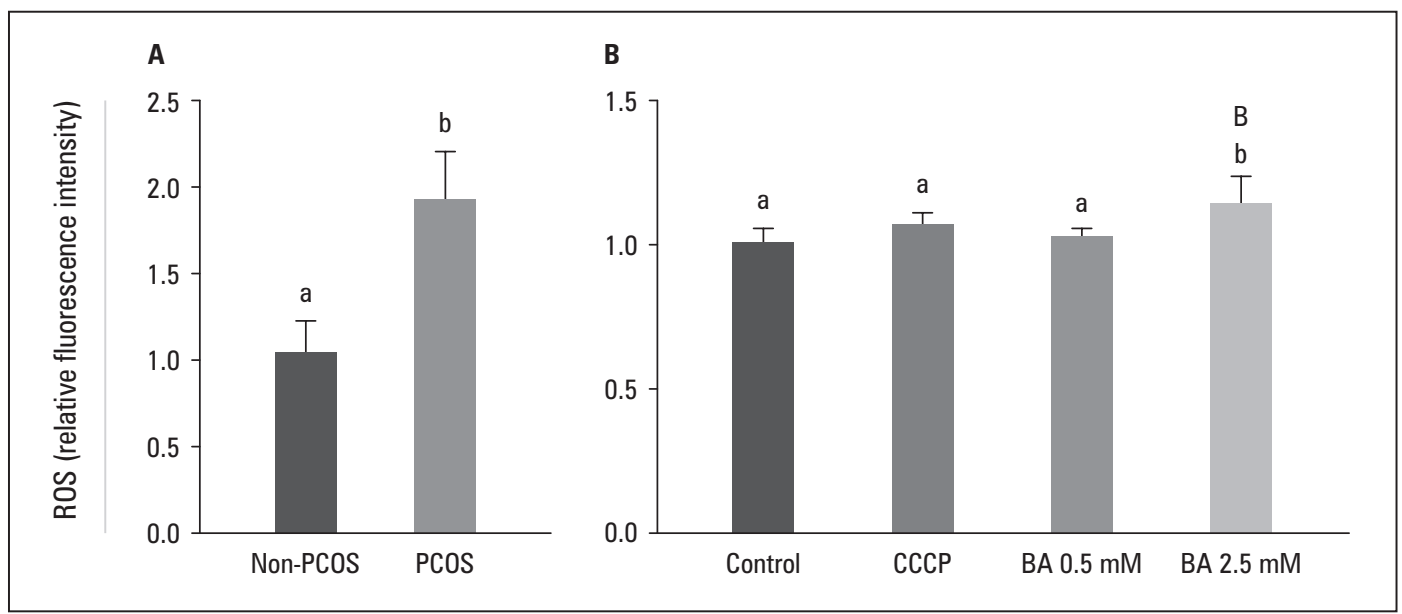

Figure 4. Amount of reactive oxygen species (ROS) in human granulosa cells (GCs) from patients without polycystic ovary syndrome (non-PCOS) and patients with polycystic ovary syndrome (PCOS), following the treatment with inhibitors of mitochondrial function or glycolysis activity. GCs were treated with CCCP $(10 \mathrm{mM})$ and BA $(0.5 \mathrm{mM} / 2.5 \mathrm{mM})$, respectively, for 30 min, and the amount of ROS was then measured by fluorescence spectrophotometer. Average fluorescence intensity of human GCs was defined as 1.0. Fluorescence intensity of ROS was normalised by control in $2 \times 10^{4}$ GCs. The t-test and one-way ANOVA were used for statistical analysis ( $a-b$, $p<0.05)$

of GCs with mitochondrial inhibitor CCCP and glycolysis inhibitor BA, the ATP content was significantly reduced in all GCs (control, $8.775 \pm 0.28 \mu \mathrm{m}$; CCCP $10 \mu \mathrm{M}, 8.116 \pm 0.33 \mu \mathrm{m}$; BA $0.5 \mathrm{mM}, 1.863 \pm 0.27 \mu \mathrm{m}$; BA $2.5 \mathrm{mM}, 0.482 \pm 0.16 \mu \mathrm{m} ; \mathrm{p}<0.05)$. Furthermore, the glycolysis inhibitor had a more pronounced inhibition effect on GCs, when compared with mitochondrial inhibitors, and the inhibitory effect of BA $2.5 \mathrm{mM}$ was stronger than that of BA $0.5 \mathrm{mM}$. Furthermore, the mitochondrial respiratory chain played critical roles in cell function and energy metabolism, while the glycolysis had the same or even stronger effect in the energy metabolism of GCs.

\section{Reactive oxygen species levels}

Compared with the non-PCOS group, the ROS levels of GCs obtained from PCOS significantly increased (1.9-fold). The investigators previously reported that CCCP $(10 \mu \mathrm{M})$ and BA $(0.5 \mathrm{mM} / 2.5 \mathrm{mM})$ both affect mitochondrial function in GCs and induce the decrease of MMP and ATP content. Meanwhile, the intracellular ROS generation in GCs increases, accompanied by the former decline tendency. Furthermore, $10 \mu \mathrm{M}$ of CCCP and $0.5 \mathrm{mM}$ of BA slightly increased the ROS levels, but the difference was not statistically significant. The treatment with $2.5 \mathrm{mM}$ of BA obviously enhanced the ROS level of GCs by 1.15-fold ( $\mathrm{p}<0.05$, Fig. 4). Therefore, glycolysis not only plays a critical role in GC energy metabolism, but also influences mitochondrial oxidative metabolism.

\section{The mitochondrial DNA copy number in GCs and oxidative stress markers in FF}

The relative mtDNA levels determined by real-time Q-PCR were significantly downregulated in GCs obtained from PCOS patients $(1.021 \pm 0.06$ vs. $0.761 \pm 0.05$, $\mathrm{p}=0.0018$; Fig. 5A). Compared with the non-PCOS group, the levels of MDA, SOD, and 8-OHDG in FF detected via correlative experiment kits significantly increased, which was accompanied by a decrease in mtDNA level in PCOS patients (MDA, $1.558 \pm 0.10$ vs. $2.047 \pm 0.23 \mathrm{nmol} / \mathrm{mL}$; SOD, $58.71 \pm 3.13 v s .71 .17 \pm 5.03$ $\mathrm{U} / \mathrm{mL}$; 8-OHDG, $0.7163 \pm 0.06$ vs. $0.9469 \pm 0.10 \mathrm{ng} / \mathrm{mL}$; Fig. 5B-D). The increase in peroxidation injuries that index MDA and 8-OHDG were accompanied by compensatory elevated antioxidant damage index SOD.

\section{Expression of the glycolysis-associated gene and HIF-1 $\alpha$ in GCs, and glucose metabolism in FF} In order to determine whether the glycolysis-associated gene expression in GCs is correlated with follicle development, the GLUT1/LDHA/PFKP gene expression levels in non-PCOS and PCOS patients were measured. Compared to non-PCOS, the mRNA expression levels of GLUT1/LDHA/PFKP were significantly downregulated in GCs obtained from PCOS patients (GLUT1, $1.077 \pm 0.10$ vs. $0.734 \pm 0.10 ;$ LDHA, $1.005 \pm 0.04$ vs. $0.641 \pm 0.1, \mathrm{PFKP}, 1.103 \pm 0.08$ vs. $0.723 \pm 0.10$; Fig. 6A-C). Collectively, these data suggest that in ovarian PCOS, the expression levels of glycolytic rate-limiting enzymes in GCs were downregulated. 


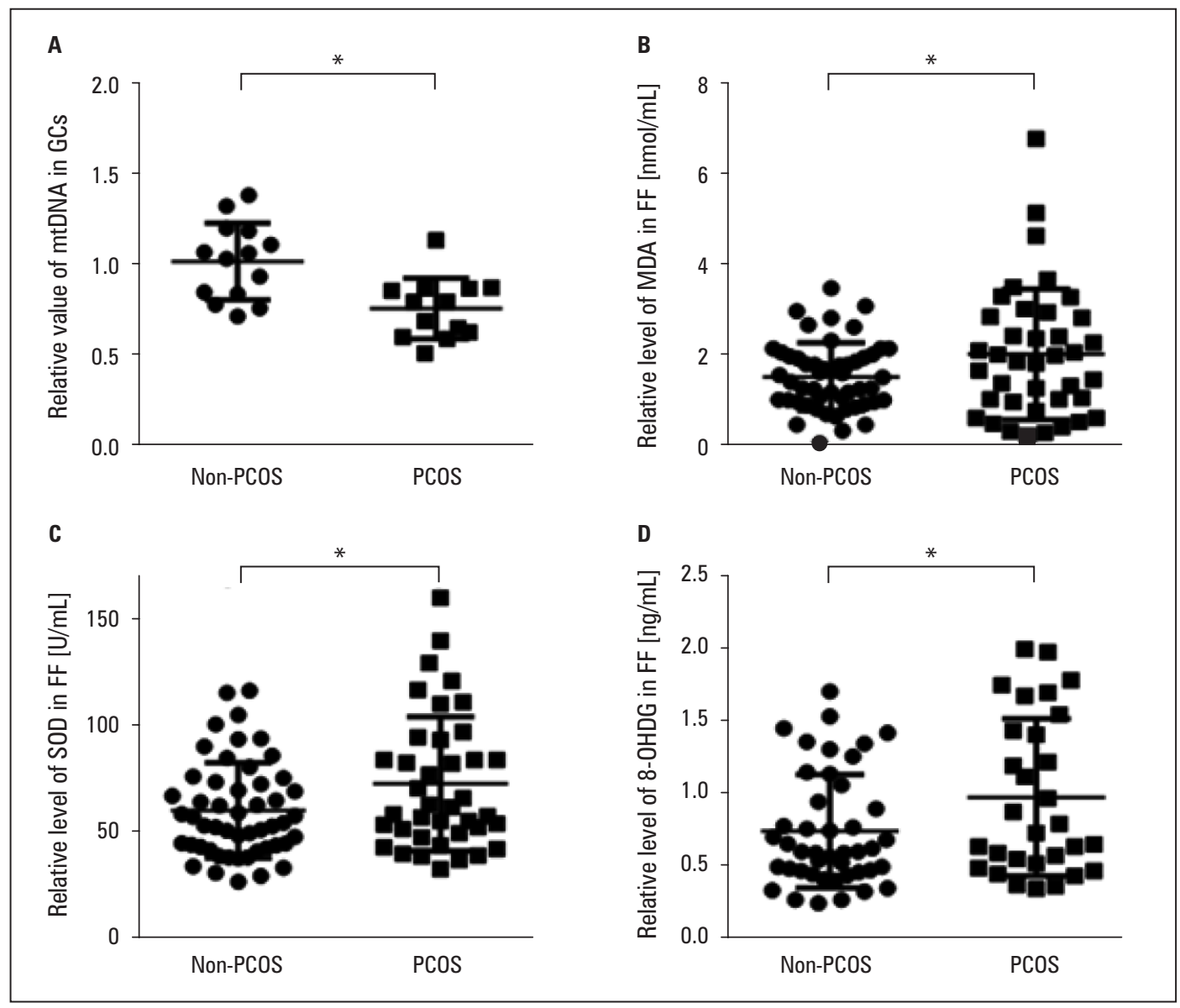

Figure 5. The mitochondrial DNA (mtDNA) copy number in in human granulosa cells (GCs) and the status of peroxidation and antioxidant in FF from patients without polycystic ovary syndrome (non-PCOS) and patients with polycystic ovary syndrome (PCOS). A. The relative level of $m t D N A$ copy number in GCs of PCOS and non-PCOS were measured by real-time qPCR. B-D. Imbalances of abnormal peroxidation and antioxidant status in follicular fluid (FF). The level of malondialdehyde (MDA), superoxide dismutase (SOD), and 8-hydroxydeoxyguanosine (8-OHDG) in FF were detected via correlative experiment kits, respectively. Data are presented as mean \pm SEM. The t-test was used for statistical analysis $\left({ }^{*} p<0.05\right)$

After GC isolation, the glucose levels in FF were measured, and the glucose uptake level was evaluated. Similar to the low expression pattern of the glycolysis-associated gene in GCs obtained from PCOS, the levels of elevated glucose $(2.812 \pm 0.33$ vs. $5.505 \pm 0.51 \mathrm{mmol} / \mathrm{L}$, Fig. 6D) were accompanied by the levels of decreased pyruvate $(0.2347 \pm 0.03$ vs. $0.1673 \pm 0.01 \mu \mathrm{mol} / \mathrm{mL}$, Fig. 6E). These results suggest that the downregulation of glycolysis-associated gene expression is a frequent occurrence in patients with PCOS, because the glycolysis dysfunction might impair the folliculogenesis in PCOS.

HIF- $1 \alpha$ plays a key role in ovarian follicular development and ovulation. In order to determine whether the HIF- $1 \alpha$ gene takes part in the pathophysiological changes in the ovaries of women with PCOS, the HIF-1 $\alpha$ gene expression levels in non-PCOS and PCOS patients were measured. Compared to non-PCOS patients, the mRNA expression levels of HIF-1 $\alpha$ were significantly reduced in GCs obtained from PCOS patients $(1.141 \pm 0.11$ vs. $0.707 \pm 0.09$, Fig. 6 F). HIF- $1 \alpha$ can be largely increased in developing follicles, and the remarkable upregulation of HIF-1 $\alpha$ triggers the cell glucose uptake. In the present study, consistent with the downregulated HIF-1 $\alpha$ in GCs, the mRNA expression levels of GLUT1, LDHA, and PFKP decreased in GCs obtained from PCOS patients.

\section{CCCP-induced HIF-1 $\alpha$ promotes glycolysis- -associated gene expression and regulates glycolysis and cell viability}

In order to further demonstrate the energy transformation function and mechanism in GCs under low oxygen conditions, the mitochondrial dysfunction model was applied. In the presence of $10 \mu \mathrm{M}$ of CCCP in GCs, the mitochondrial respiratory chain was impaired, the oxygen-dependent degradation of HIF- $1 \alpha$ was inhibited, and HIF- $1 \alpha$ accumulation increased by 1.8 -fold (Fig. 7A). Furthermore, the mRNA expression levels of 


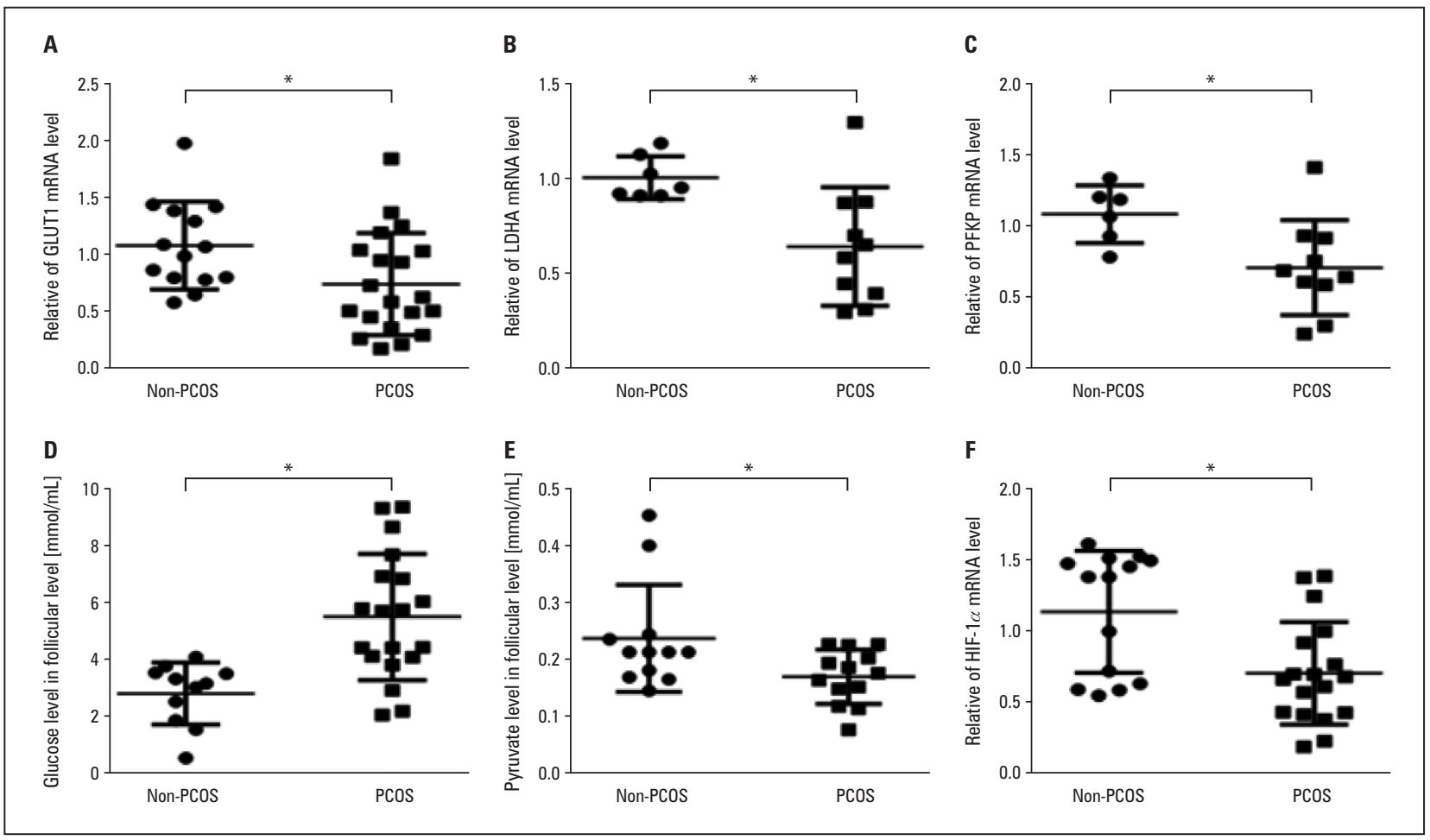

Figure 6. The expression of glycolysis-associated gene and HIF-1 $\alpha$ in granulosa cells (GCs) and glucose metabolism in follicular fluid (FF) from patients without polycystic ovary syndrome (non-PCOS) and patients with polycystic ovary syndrome (PCOS). A-D. The relative mRNA levels of GLUT1, LDHA, PFKP, and HIF-1 $\alpha$ in GCS from PCOS and non-PCOS were measured by real-time qPCR. E-F. Abnormal glucose metabolism in follicular fluid (FF). Data are presented as mean \pm SEM. Student's $t$-test was used for statistical analysis $\left({ }^{*} p<0.05\right)$

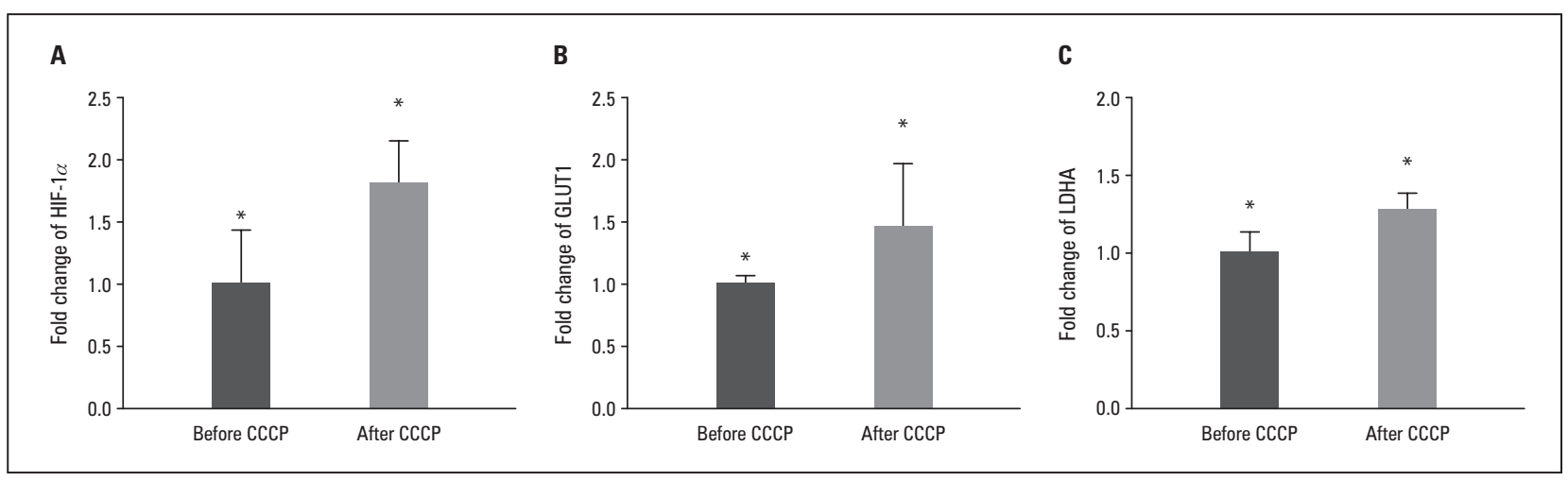

Figure 7. The expression of glycolysis-associated gene in human granulosa cells (GCs), following the treatment with inhibitors of mitochondrial function. A-C. The relative mRNA levels of HIF-1 $\alpha$, GLUT1, and LDHA in GCs treated with $10 \mu M$ carbonyl cyanide m-chlorophenyl hydrazine (CCCP) were measured by real-time qPCR. Data are presented as mean \pm SEM. Student's t-test was used for statistical analysis $\left({ }^{*} p<0.05\right)$

GLUT1 and LDHA in GCs were upregulated, which in turn increased the glucose transport and uptake. In addition, GLUT1 was upregulated 1.4-fold (Fig. 7B), while LDHA increased 1.2-fold (Fig. 7C). Consistent with the expression patterns of the HIF-1 $\alpha$, GLUT1, and LDHA genes, glucose consumption gradually increased after 24,48 , and 72 hours of culturing GCs after CCCP treatment, and the viability of cells tested by CCK- 8 also increased (Fig. 8A-B, p < 0.05). These results suggest that ATP production via the mitochondria was inhibited, and switching of the energy metabolism to the glycolytic pathway was initiated. 


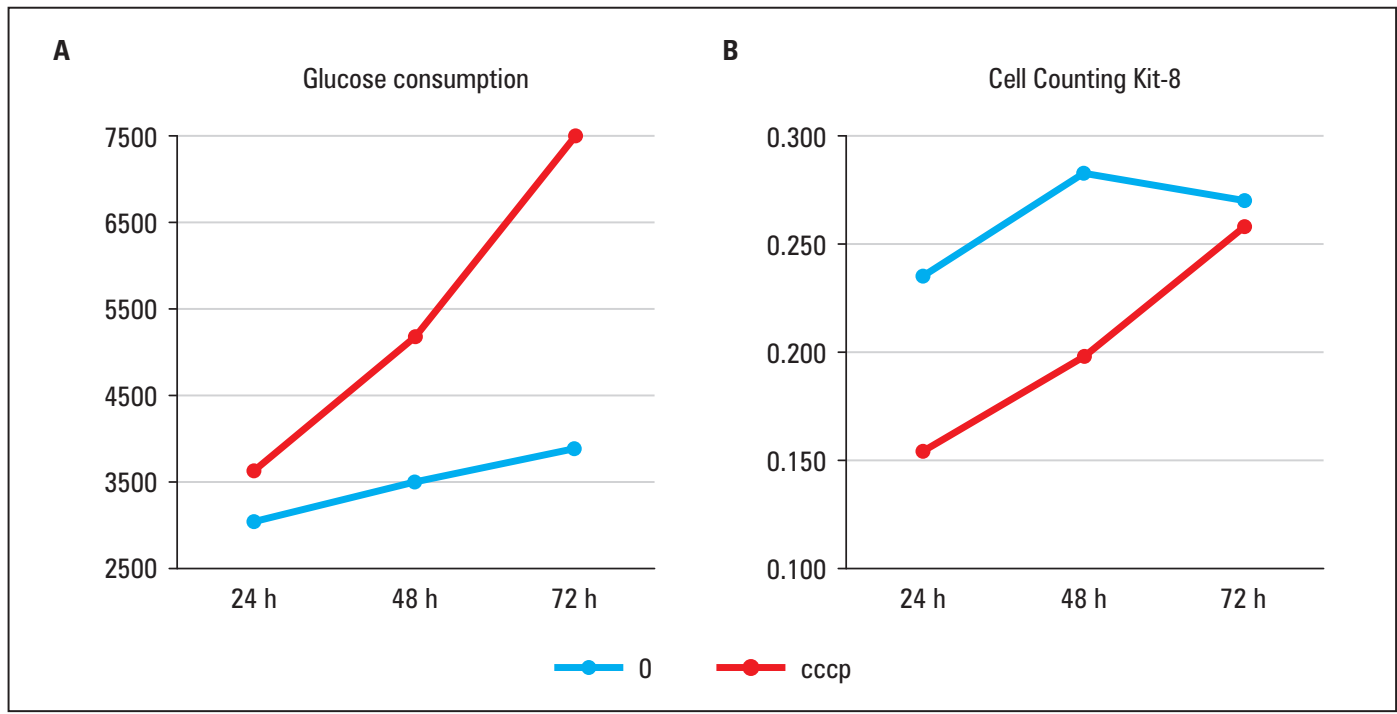

Figure 8. The level of glucose consumption of human granulosa cells (GCs) and cell viability, following the treatment with inhibitors of mitochondrial function. A. The level of glucose consumption of GCs after $10 \mu \mathrm{M}$ carbonyl cyanide m-chlorophenyl hydrazine (CCCP) treatment at $24 h, 48 h$, and $72 \mathrm{~h}$ time points was detected by glucose kit. B. Cell Counting Kit-8 (CCK8) confirmed the cell growth after $10 \mu M$ CCCP treatment at $24 h, 48 h$, and $72 \mathrm{~h}$ time points. Data are presented as mean \pm SEM. Student's t-test was used for statistical analysis $\left({ }^{*} p<0.05\right)$

\section{Discussion}

In the present study, it was confirmed that there was excessive glucose in the follicle and serum obtained from patients with PCOS. In addition to the changes in biochemical indicators, decreased glycolysis activity, which was indicated by the reduced expression of PFKP, GLUT1, and LDHA, was also observed in GCs obtained from PCOS patients.

Animal studies on cows have demonstrated that the metabolic change from OXPHOS to glycolysis in folliculogenesis under low oxygen levels plays a key role in rapid GC proliferation [16]. In the present study, it was demonstrated that mitochondrial inhibitors and the glycolysis inhibitor jointly participated in GC energy factory works, and mitochondrial function, which included MMP and ATP levels, significantly declined, and this was accompanied by increased ROS production in GCs of PCOS. The source of excessive ROS generation in PCOS might be due to the impaired mitochondrial OXPHOS in GCs. It was also found that glycolysis not only plays a critical role in GC energy metabolism, but also influences mitochondrial oxidative metabolism.

Furthermore, it was also found the mitochondrial DNA (mtDNA) genes of GCs were downregulated in PCOS patients. It has been shown that the maintenance of the mtDNA copy number is essential for the preservation of mitochondrial function and cell growth. These results suggest that the copy number alteration of mitochondria DNA in GCs in PCOS patients were accompanied by mitochondrial oxidative injuries, thereby resulting in the higher MDA levels and 8-OHDG levels. Abnormal mitochondrial function at the cellular level can affect whole-body metabolic homeostasis [4]. A reduced mtDNA copy number is important for abnormal peroxidation and antioxidant status in FF of PCOS. These data are in line with previous studies, which revealed that OS level significantly increases in patients with PCOS, which compared with non-PCOS, which oxidative status is evaluated by circulating markers, such as malondialdehyde (MDA), superoxide dismutase (SOD), and glutathione peroxidase. Mitochondria play a vital role in cellular energy production and energy metabolism. The present study investigated the mitochondrial function and glycolysis activity in PCOS patient-derived GCs, and it was considered that the low expression of mtDNA and low expression levels of HIF1- $\alpha$, GLUT1, LDHA and PFKP are causal factors of the suboptimal conditions for the process of GC differentiation during follicle development, which in turn affects oocyte maturation and induces abnormal oocyte quality and embryonic development. GC manifest two energy metabolism pathways during the late stage of follicle development and maturation, and the glycolysis has a greater impact on the growth of follicles.

The decreased mtDNA content in peripheral leukocytes was associated with the development of type 2 diabetes mellitus. However, it remains unclear whether mitochondrial abnormalities are a primary defect in the energy change of PCOS. In order to explore the mechanisms underlying the pathogenesis of 
metabolic changes in GCs, the role of mitochondrial inhibitor CCCP on energy metabolism of GCs was explored. After the CCCP treatment, the mitochondrial respiratory chains were impaired, and the balance of oxygen pressure in the follicle microenvironment was broken, which in turn stimulated HIF-1 $\alpha$, GLUT1, LDHA, and PFKP expression, promoted glucose consumption, and increased the viability of GCs. In PCOS patients, the damaged mitochondrial function and glycolysis showed that abnormal energy metabolic not only affects GC proliferation, but may also disturb the metabolic switch of energy from OXPHOS to glycolysis in GCs during follicle growth and development. Specifically, the reduction of mitochondrial activity in PCOS might inhibit the HIF-1 $\alpha$ signalling, thereby further retarding the switch of energy metabolism during follicular growth. These results suggest that the downregulation of glycolysis-associated gene expression is a frequent occurrence in patients with PCOS, because glycolysis dysfunction might impair folliculogenesis in PCOS. Furthermore, on the basis of comparing the gene expression between non-PCOS and PCOS in matured GCs, it was considered that low expression levels of GLUT1, LDHA, and PFKP in PCOS patients could be considered as causal factors of sub-optimal conditions for GC differentiation during follicle development, which in turn affects oocyte maturation. The reduced expression levels of HIF-1 $\alpha$ might be responsible for the downregulation of glycolysis in GCs in PCOS patients.

After the application of glycolytic inhibitors, the MMP and ATP of GCs significantly declined. However, in the presence of mitochondrial inhibitors, the glucose transport and uptake-associated gene expression increased. These results prove that the energy status of GCs was maintained by both the mitochondrial oxidative respiratory chain and glycolysis. In the presence of CCCP, the expression levels of HIF- $1 \alpha$ and GLUT1, LDHA, and PFKP in GCs were upregulated. Meanwhile, the glucose consumption and viability of cells was gradually elevated. These data suggest that under hypoxia conditions the normal mitochondrial function was ensured, and the adaptive transformation of metabolism pattern may exist and play an important part in follicular development. ATP production via the mitochondria was inhibited, and the switching energy metabolism to the glycolytic pathway was initiated. This transformation promotes cell viability. In summary, it was demonstrated that in PCOS, mitochondrial dysfunction not only affects the energy state of GCs, but also inhibits the energy metabolic switch from mitochondrial to glycolysis metabolism.

\section{Conclusion}

The present study revealed that abnormal mitochondrial function and disordered metabolic transformation from OXPHOS to glycolysis can be identified during folliculogenesis in PCOS patients. The failed switch of energy status can be attributed to the mitochondrial dysfunction of GCs. The improvement of mitochondrial respiratory function and glucose metabolism might enhance the competence of the embryo from PCOS. Further studies would be conducted on the important role of the HIF pathway in follicular development, which mainly depends on the normal mitochondrial function in GCs.

\section{References}

1. McCartney C, Marshall J. Polycystic Ovary Syndrome. N Engl J Med. 2016; 375(1): 54-64, doi: 10.1056/nejmcp1514916, indexed in Pubmed: 27406348

2. Qiao J. Pay more attention to ethnic differences in polycystic ovary syndrome phenotypic expression. Chin Med J (Engl). 2013; 126(11): 2003-2006, indexed in Pubmed: 23769547.

3. Sirmans SM, Pate KA. Epidemiology, diagnosis, and management of polycystic ovary syndrome. Clin Epidemiol. 2013; 6: 1-13, doi: 10.2147/CLEP.S37559, indexed in Pubmed: 24379699.

4. Qiao J, Feng HL. Extra- and intra-ovarian factors in polycystic ovary syndrome: impact on oocyte maturation and embryo developmental competence. Hum Reprod Update. 2011; 17(1): 17-33, doi: 10.1093/humupd/dmq032, indexed in Pubmed: 20639519.

5. Heijnen EM, Eijkemans MJC, Hughes EG, et al. A meta-analysis of outcomes of conventional IVF in women with polycystic ovary syndrome. Hum Reprod Update. 2006; 12(1): 13-21, doi: 10.1093/humupd/dmi036, indexed in Pubmed: 16123051.

6. Vartanyan EV, Tsaturova KA, Devyatova EA, et al. Improvement in quality of oocytes in polycystic ovarian syndrome in programs of in vitro fertilization. Gynecol Endocrinol. 2017; 33(sup1): 8-11, doi: 10.1080/095 13590.2017.1399699, indexed in Pubmed: 29264980.

7. Fair T. Mammalian oocyte development: checkpoints for competence. Reprod Fertil Dev. 2010; 22(1): 13-20, doi: 10.1071/RD09216, indexed in Pubmed: 20003841

8. Franks S, McCarthy MI, Hardy K. Development of polycystic ovary syndrome: involvement of genetic and environmental factors. Int J Androl. 2006; 29(1): 278-85; discussion 286, doi: 10.1111/j.1365-2605.2005.00623.x , indexed in Pubmed: 16390494.

9. Chronowska E. High-throughput analysis of ovarian granulosa cell transcriptome. Biomed Res Int. 2014; 2014: 213570, doi: 10.1155/2014/213570, indexed in Pubmed: 24711992.

10. Combelles CMH, Holick EA, Paolella LJ, et al. Profiling of superoxide dismutase isoenzymes in compartments of the developing bovine antral follicles. Reproduction. 2010; 139(5): 871-881, doi: 10.1530/REP-09-0390, indexed in Pubmed: 20197373.

11. Sutton-McDowall ML, Gilchrist RB, Thompson JG. The pivotal role of glucose metabolism in determining oocyte developmental competence. Reproduction. 2010; 139(4): 685-695, doi: 10.1530/REP-09-0345, indexed in Pubmed: 20089664.

12. Downs SM, Mosey JL, Klinger J. Fatty acid oxidation and meiotic resumption in mouse oocytes. Mol Reprod Dev. 2009; 76(9): 844-853, doi: 10.1002/mrd.21047, indexed in Pubmed: 19455666.

13. Wu LLY, Dunning KR, Yang $X$, et al. High-fat diet causes lipotoxicity responses in cumulus-oocyte complexes and decreased fertilization rates. Endocrinology. 2010; 151(11): 5438-5445, doi: 10.1210/en.2010-0551, indexed in Pubmed: 20861227.

14. Yang X, Wu LL, Chura LR, et al. Exposure to lipid-rich follicular fluid is associated with endoplasmic reticulum stress and impaired oocyte maturation in cumulus-oocyte complexes. Fertil Steril. 2012; 97(6): 1438-1443, doi: 10.1016/j.fertnstert.2012.02.034, indexed in Pubmed: 22440252

15. Jakimiuk AJ, Weitsman SR, Navab A, et al. Luteinizing hormone receptor, steroidogenesis acute regulatory protein, and steroidogenic enzyme messenger ribonucleic acids are overexpressed in thecal and granulosa cells from polycystic ovaries. J Clin Endocrinol Metab. 2001; 86(3): 1318-1323, doi: 10.1210/jcem.86.3.7318, indexed in Pubmed: 11238527. 
Shiratsuki S, Hara T, Munakata Y, et al. Low oxygen level increases proliferation and metabolic changes in bovine granulosa cells. Mol Cell Endocrinol. 2016; 437: 75-85, doi: 10.1016/j.mce.2016.08.010, indexed in Pubmed: 27519633.

16. Stefano GB, Kream RM. Glycolytic Coupling to Mitochondrial Energy Production Ensures Survival in an Oxygen Rich Environment. Med Sci Monit. 2016; 22: 2571-2575, doi: 10.12659/msm.899610, indexed in Pubmed: 27439008 .

17. Stark H, Fichtner M, König R, et al. Causes of upregulation of glycolysis in lymphocytes upon stimulation. A comparison with other cell types. Biochimie. 2015; 118: 185-194, doi: 10.1016/j.biochi.2015.09.017, indexed in Pubmed: 26382968.
18. Laganà AS, Rossetti $\mathrm{P}$, Buscema $\mathrm{M}$, et al Metabolism and Ovarian Function in PCOS Women: A Therapeutic Approach with Inositols. In J Endocrinol. 2016; 2016: 6306410, doi: 10.1155/2016/6306410, indexed in Pubmed: 27579037.

19. González F, Rote NS, Minium J, et al. Reactive oxygen species-induced oxidative stress in the development of insulin resistance and hyperandrogenism in polycystic ovary syndrome. J Clin Endocrinol Metab. 2006; 91(1): 336-340, doi: 10.1210/jc.2005-1696, indexed in Pubmed: 16249279

20. Reddy TV, Govatati S, Deenadayal M, et al. Impact of mitochondrial DNA copy number and displacement loop alterations on polycystic ovary syndrome risk in south Indian women. Mitochondrion. 2019, 44: 35-40, doi: 10.1016/j.mito.2017.12.010, indexed in Pubmed: 29278759 